\title{
Simulating the Zody Emission in the Planck Mission
}

\author{
Michele Maris* \\ INAF-Osservatorio Astronomico di Trieste \\ Via G.B.Tiepolo 11, I-34131 Trieste, Italy \\ E-mail: mariseoats.inaf.it
}

\section{Carlo Burigana}

INAF-IASF Bologna

Via Gobetti 101, I-40129 Bologna, Italy

E-mail: burigana@iasfbo.inaf.it

\section{Sandro Fogliani}

INAF-Osservatorio Astronomico di Trieste Via G.B.Tiepolo 11, I-34131 Trieste, Italy

E-mail: fogliani@oats.inaf.it

\begin{abstract}
The increasing sensitivity of cosmic microwave background (CMB) missions will require to significantly improve the accuracy in the subtraction of the various sources of Galactic foreground, from the most relevant components (synchrotron, dust and free-free emission) to those usually considered of minor relevance in CMB experiments. With respect to other Galactic diffuse components, the Zodiacal Light Emission (ZLE) is peculiar, depending not only on the observing direction but also on the location of the observer within the Solar System: ZLE behaves then as a large scale, time-dependent foreground. Starting from the existing far-infrared ZLE models, we discuss the impact of ZLE contribution in CMB maps and the level of contamination in time ordered data and maps expected from the forthcoming PLANCK space mission as well as the PLANCK capability to increase our knowledge of the ZLE properties.
\end{abstract}

$C M B$ and Physics of the Early Universe 20-22 April 2006

Ischia, Italy

\footnotetext{
* Speaker.
} 


\section{Introduction}

The increasing sensitivity of cosmic microwave background (CMB) missions will require a careful subtraction of any source of weak "Galactic" foreground, asking to consider in CMB experiments other sources of Galactic foregrounds over the most relevant ones, i.e. synchrotron, dust, and free-free emission. In this context, the Zodiacal Light Emission (ZLE) due to the thermal emission from the cloud of Interplanetary Dust Particles (IDPs) permeating the Solar System represents a significant component. At frequencies $\approx 10 \mathrm{THz}$ the ZLE dominates the sky emissivity, and in preparing templates of Galactic emission from observations at these frequencies the contamination of ZLE has to be accurately accounted for, since the ability to model and remove the ZLE will largely fix the final accuracy of the Galactic templates. At frequencies below $\approx 1 \mathrm{THz}$ the ZLE is subdominant compared to the Galaxy, but its surface brightness is still significant particularly in regions where the Galaxy emission is weak [1,2]. It has also to be considered that the ZLE does not depend only on the instrument pointing direction, $\mathbf{P}$, but also on the position of the observer, $\mathbf{R}_{\mathrm{P}}$, within the Solar System. Then, the ZLE behaves as a time-dependent foreground and, when not properly removed, introduces subtle systematic effects.

\section{Model and extrapolations}

As for the Galactic dust contamination, the modelling of the ZLE has to be based on farinfrared observations, at least in what regard the geometrical aspects of the IDPs distribution. Key data for the ZLE below $300 \mu \mathrm{m}$ have been obtained by IRAS [3], COBE [1, 4] and ISO [5, 6]. The starting point for this analysis is the COBE/DIRBE model for the ZLE which describes the expected local 3D emissivity within the IDPs complex [4]. In particular, among the various components in which the IDPs are distributed we refer here to the dominant Smooth component which accounts for more than $90 \%$ of the ZLE. Then, for frequencies $f \lesssim 1 \mathrm{THz}$ the total brightness of the ZLE integrated along a given line-of-sight is

$$
I_{f}\left(\mathbf{P}, \mathbf{R}_{\mathbf{P}}\right)=E_{f} Z_{f}\left(\mathbf{P}, \mathbf{R}_{\mathbf{P}}\right),
$$

where $Z_{f}\left(\mathbf{P}, \mathbf{R}_{\mathrm{P}}\right)$ gives the spatial dependence and the Emissivity Factor $E_{f}$ is a correction with respect to a pure blackbody emission law, related to the composition and size distribution of dust grains. Following [4] it is assumed $E_{f}=1$ for $f=12 \mathrm{THz}$. The spatial dependence is given by the integral along the line-of-sight

$$
Z_{f}\left(\mathbf{P}, \mathbf{R}_{\mathbf{P}}\right)=\int_{0}^{+\infty} d s N\left(\mathbf{R}_{\mathbf{P}}+s \mathbf{P}\right) B_{f}\left(T\left(\mathbf{R}_{\mathrm{P}}+s \mathbf{P}\right)\right),
$$

where $s$ is the distance from the observer along $\mathbf{P}, B_{f}(T)$ the blackbody brightness, $N(\mathbf{r})$ the dust density at a given location in the Solar System (assuming $N(\mathbf{r}) \equiv 0$ for $|\mathbf{r}|>5.2 \mathrm{AU}), T(\mathbf{r})$ the local dust temperature, assumed to scale as $|\mathbf{r}|^{-0.467}$.

In long duration $\mathrm{CMB}$ experiments the sky is observed while the observer is orbiting around the Sun and consequently the ZLE will show seasonal modulations at the level of 5-15\% [2]. Changes in the scanning strategy or observation epoch will result in different realizations of ZLE sky maps even when the same set of pointing directions is taken. To condensate into a statical map 


\begin{tabular}{rcccc}
\hline \hline & \multicolumn{4}{c}{ Frequency Channell } \\
& $217 \mathrm{GHz}$ & $353 \mathrm{GHz}$ & $545 \mathrm{GHz}$ & $857 \mathrm{GHz}$ \\
\hline$E_{f}$ & $4 \times 10^{-2}$ & $1 \times 10^{-1}$ & $3 \times 10^{-1}$ & $6 \times 10^{-1}$ \\
$\min I_{f}[\mathrm{MJy} / \mathrm{sr}]$ & $5 \times 10^{-4}$ & $4 \times 10^{-3}$ & $2 \times 10^{-2}$ & $1 \times 10^{-1}$ \\
$\operatorname{mean} I_{f}[\mathrm{MJy} / \mathrm{sr}]$ & $1 \times 10^{-3}$ & $8 \times 10^{-3}$ & $5 \times 10^{-2}$ & $3 \times 10^{-1}$ \\
$\max I_{f}[\mathrm{MJy} / \mathrm{sr}]$ & $2 \times 10^{-3}$ & $2 \times 10^{-2}$ & $1 \times 10^{-1}$ & $6 \times 10^{-1}$ \\
\hline \hline
\end{tabular}

Table 1: Predicted values of $E_{f}$ and of the ZLE at the highest PLANCK frequencies. The table reports minimum, mean and maximum ZLE within a $\approx 50 \%$ uncertainty for a one year mission.

this dynamical information we exploit the cylindrical symmetry of COBE distribution of the IDPs Smooth component. We develop a serie expansion of $Z_{f}\left(\mathbf{P}, \mathbf{R}_{\mathbf{P}}\right)$ about an averaged orbit in the IDPs cloud reference frame [2]. This is equivalent to calculate a kernel map for $Z_{f}\left(\mathbf{P}, \mathbf{R}_{\mathrm{P}}\right)$ which is good for a given "nominal" mission together with coefficients to be used in computing variations of the map for a range of possible variations in the mission. This method allows at the same time the generation of data streams of ZLE signals for a given list of spacecraft positions and pointing directions, and the generation of specialized time averaged maps (e.g. yearly averaged maps) for a specific mission, orbit and scanning strategy ${ }^{1}$.

The extrapolation of $E_{f}$ at frequencies below $1 \mathrm{THz}$ is a more delicate problem. COBE/DIRBE measures extend down to $f=1.2 \mathrm{THz}$, which fixes the lowest frequency for which the COBE model provides values of $E_{f}$ [4]. However COBE/FIRAS provides measures of ZLE averaged over the sky and over one year down-to $f \approx 3 \times 10^{2} \mathrm{GHz}$ [1] but with a not high $\mathrm{S} / \mathrm{N}$ ratio. We then compare the COBE/DIRBE measures with simulated yearly and full-sky averaged values of $Z_{f}\left(\mathbf{P}, \mathbf{R}_{\mathrm{P}}\right)$ in order to evaluate $E_{f}$. In this way we can obtain numerical estimates for these parameters [2]. Similar results are also obtained directly extrapolating $E_{f}$ values derived by COBE/DIRBE at $f<12 \mathrm{THz}$ down to the required frequencies. Tab. 1 summarizes the results of these calculations giving the minimal and maximal yearly averaged levels of contamination at frequencies relevant for the PLANCK mission.

The left frame of Fig. 1 represents a typical realization of a ZLE signal at $857 \mathrm{GHz}$ (together with secondary components and uncertainties) compared to the Galaxy. The right frame shows the relative contribution of ZLE brighness and instrumental noise respect to the Galaxy. In the plot the ZLE and the noise contributions are averaged over a circular band of about $85^{\circ}$ of radius and drawn around an axis of given ecliptical longitude. The same is done for the Galaxy. The red band is the noise, the white line the expected ZLE over Galaxy averaged ratio, the blue band the $\pm 1 \sigma$ band, the yellow line the maximum ratio. The plotted values are ordered as function of the ecliptical longitude of spin axis vector. The data are calculated for patches of $1^{\circ}$ in radius. The noise is referred to a 14 month mission (2 sky surveys).

\footnotetext{
${ }^{1}$ Tables of serie expansions of ZLE for all of the PLANCK frequencies, the related software in IDL, and documentation may be required to the authors.
} 

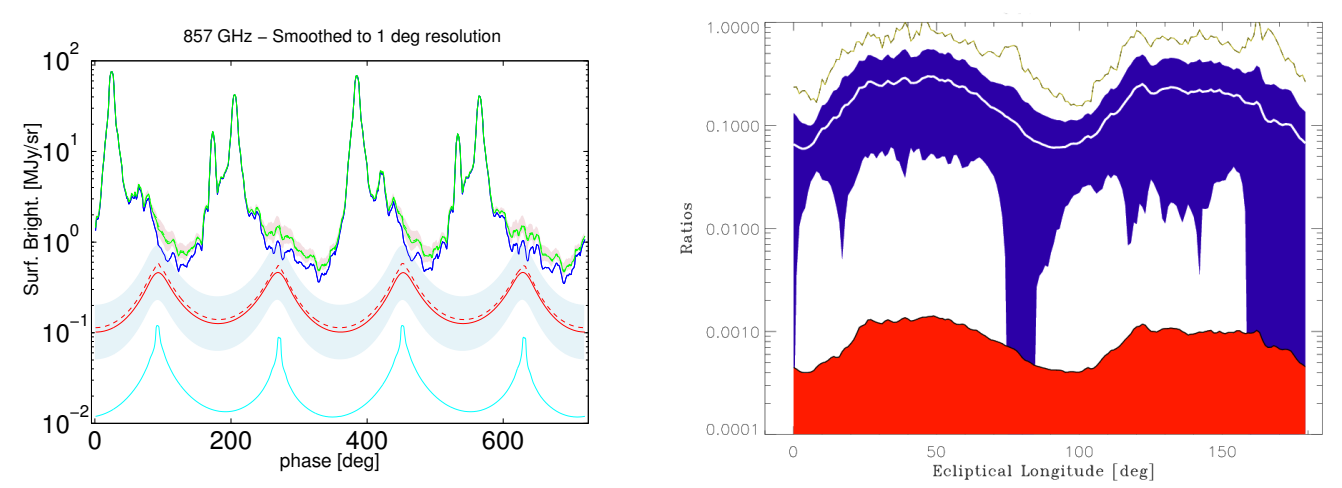

Figure 1: Left panel: simulated data stream of surface brightnesses (MJy/sr) expected at $857 \mathrm{GHz}$ for the ZLE - smooth component (red), secondary components (cyan) and uncertainties in the prediction (bands) the Galaxy (green), and the sum of ZLE and Galaxy (blue). Also signals from ZLE (red-dashed) as after summation of the Galaxy (green dashed) are shown. The ordinate is the phase along the scan circle. Right panel: relative averaged contribution of ZLE, and noise compared to the averaged Galaxy and computed over circular bands, as a function of the ecliptical longitude of the spin axis vector.

\section{Detection and removal of the $Z \mathbf{L E}$}

It is evident that current information does not allow to reach an accuracy better then $\approx 20 \%$ in the ZLE removal; this is due in particular to the uncertainties on $E_{f}$ below $1 \mathrm{THz}$. New, direct measures are necessary to improve the situation. The ability of PLANCK to detect the ZLE signal improving the accuracy in $E_{f}$ determination has been analyzed in [2] assuming that the COBE model properly represents the spatial distribution of the ZLE leaving as a free parameter $E_{f}$. Two methods have been considered, the first based on the comparison of a template map for the Galactic emission with a spatial template for the ZLE calculated for given mission orbit and scanning strategy, the second method is based on the comparison of observations of the same regions of sky taken at different epochs then exploiting the seasonal dependence in the ZLE observation. In both cases, since the ZLE varies over scales of $\approx 10^{\circ}$, one can consider template maps and observations at resolutions of $\approx 1^{\circ}-2^{\circ}$ (this alleviate also the contribution of local features, as weak point sources or regions with peculiar frequency dependencies). In this kinds of analysis, it is important to apply cuts to the data excluding regions where the Galaxy is very bright. Simulations shows a typical absolute RMS uncertainty on $E_{f}$ determination induced by the limited instrumental sensitivity of $\sim 10^{-3}, 2.1 \times \sim 10^{-3}$ and $2.6 \times \sim 10^{-3}$ at $857 \mathrm{GHz}, 545 \mathrm{GHz}$ and $353 \mathrm{GHz}$, respectively. For typical expected values of $E_{f}(\approx 0.65,0.26,0.11$ for $857 \mathrm{GHz}, 545 \mathrm{GHz}, 353 \mathrm{GHz})$ the PLANCK sensitivity will allow an $E_{f}$ recovery at $0.15 \%, 0.8 \%$ and $2.4 \%(1 \sigma)$ accuracy at $857 \mathrm{GHz}, 545 \mathrm{GHz}$ and $353 \mathrm{GHz}$, respectively. Of the most relevant systematic effects, pointing and sampling uncertainty, aberration of light, Doppler shift and relative calibration uncertainty, only the last one is found to be really critical, while the errors introduced by the other ones are found to be significantly below the noise. Then, relative calibration could ultimately determine the final accuracy in the ZLE extraction from PLANCK data. For a relative calibration RMS error of $\sim 1 \%(0.1 \%)$ on patches of $2^{\circ}$ radius, we find an absolute RMS error on $E_{f}$ of $\sim 0.01-0.04(\sim 0.001-0.004)$ with only a weak dependence on the frequency in the range $\sim 300-900 \mathrm{GHz}$, corresponding to relative 
errors on $E_{f} \sim 4 \%, 10 \%, 23 \%(\sim 0.4 \%, 1 \%, 2 \%)$, respectively at $857 \mathrm{GHz}, 545 \mathrm{GHz}, 353 \mathrm{GHz}$ for the most likely $E_{f}$ values expected on the basis of COBE/FIRAS data.

\section{References}

[1] Fixsen, D.J., \& Dwek, E., 2002, Ap.J., 578, 1009

[2] M. Maris, C. Burigana, S. Fogliani, Zodiacal Light Emission in the PLANCK mission, A\&A, In press, [astro-ph/0603...].

[3] Wheelock, S.L., Gautier, T.N., Chillemi, J., Kester, D., McCallon, H., et al., 1994, IRAS Sky Survey Atlas Explanatory Supplement, JPL Pubbl. 94-11

[4] Kelsall, T., Weiland, J.T., Franz, B.A., Reach, W.T., Arendt, R.G., et al., 1998, ApJ, 508, 44 (also astro-ph/9806250)

[5] Reach, W.T., Abergel, A., Boulanger, F., Desert, F.-X., Perault, M., et al., 1996, A\&A, 315, L381

[6] Reach, W.T., Morris, P., Boulanger, F., Okumura, K., 2003, Icarus, 164, 384

[7] Bohren, C.F., \& Huffman, D. R., 1998, Absorption and Scattering of Light by Small Particles John Wiley \& Sons, ISBN: 0471293407, New York, USA 